\title{
Diagnostic value of ultrathin bronchoscopy in peripheral pulmonary lesions: a narrative review
}

\author{
Masahide Oki ${ }^{1}$, Hideo Saka ${ }^{1,2}$ \\ ${ }^{1}$ Department of Respiratory Medicine, National Hospital Organization Nagoya Medical Center, Nagoya, Japan; ${ }^{2}$ Department of Respiratory \\ Medicine, Matsunami General Hospital, Kasamatsu, Japan \\ Contributions: (I) Conception and design: All authors; (II) Administrative support: All authors; (III) Provision of study materials or patients: All \\ authors; (IV) Collection and assembly of data: All authors; (V) Data analysis and interpretation: All authors; (VI) Manuscript writing: All authors; (VII) \\ Final approval of manuscript: All authors. \\ Correspondence to: Masahide Oki, MD. Department of Respiratory Medicine, National Hospital Organization Nagoya Medical Center, Nagoya, \\ Japan. Email: masahideo@aol.com.
}

\begin{abstract}
Flexible bronchoscopes are being continuously improved, and an ultrathin bronchoscope with a working channel that allows the use of a radial-type endobronchial ultrasound (EBUS) probe is now available. The ultrathin bronchoscope has good maneuverability for passing through the small bronchi and good accessibility to peripheral lung lesions. This utility is particularly enhanced when it is used with other imaging devices, such as EBUS and navigation devices. Multimodality bronchoscopy using an ultrathin bronchoscope leads to enhanced diagnostic yield.
\end{abstract}

Keywords: Bronchoscopy; endobronchial ultrasound (EBUS); peripheral pulmonary lesions (PPLs); ultrathin bronchoscope; virtual bronchoscopic navigation (VBN)

Submitted Nov 15, 2020. Accepted for publication Dec 17, 2020.

doi: 10.21037/jtd-2020-abpd-001

View this article at: http://dx.doi.org/10.21037/jtd-2020-abpd-001

\section{Introduction}

Accurate diagnosis of small peripheral pulmonary lesions (PPLs) is still challenging (1). Bronchoscopy has been widely used for diagnosis, and instrumental and technical improvements have gradually enhanced diagnostic yield. As the bronchus branches peripherally, its diameter decreases; standard bronchoscopes with an external diameter of about $5 \mathrm{~mm}$ are too large to access the peripheral lung region. Thinner bronchoscopes have the advantage that they provide good accessibility to PPLs through small bronchi, so their use in diagnosing PPLs is reasonable. Although no formal definition has been widely accepted, we define an "ultrathin bronchoscope" as having an outer diameter $\leq 3.5 \mathrm{~mm}(2)$.

Most conventional ultrathin bronchoscopes are equipped with a working channel with an inner diameter of $1.2-\mathrm{mm}$, which allows the use of only mini-forceps $<1.2 \mathrm{~mm}$ in diameter to obtain specimens of a limited size. Therefore, despite their potential high diagnostic yield, conventional ultrathin bronchoscopes with a 1.2 -mm working channel have been regarded as an adjunct, rather than an alternative, to conventional bronchoscopes in PPL diagnosis $(3,4)$. Conventional bronchoscopy for sampling PPLs has been performed only under fluoroscopic guidance. However, some ancillary techniques, such as navigation, computed tomography (CT), and endobronchial ultrasound (EBUS), have been developed and applied to bronchoscopy. Such guided methods have increased the diagnostic yield of bronchoscopy (5). An ultrathin bronchoscope has good maneuverability when passing it through the small-airway route and good accessibility to the peripheral lung, so its utility is enhanced when combined with confirmatory tools for use when in proximity to target lesions. Several studies have demonstrated the diagnostic utility of ultrathin bronchoscopes in combination with navigation devices (6-14), CT fluoroscopy $(6,15,16)$, or cone-beam CT (CBCT) $(17,18)$. Furthermore, a next-generation ultrathin 


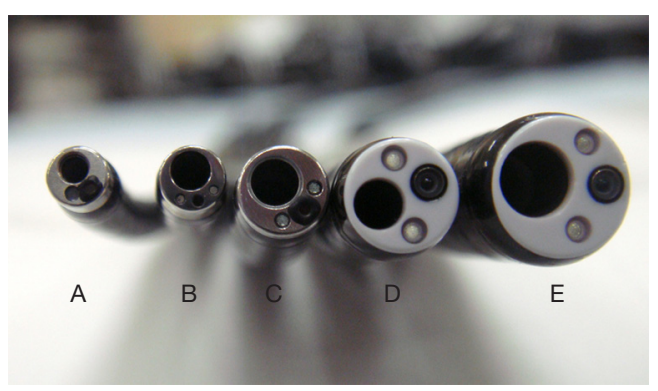

Figure 1 Flexible bronchoscopes. (A) A 2.8-mm ultrathin bronchoscope with a $1.2-\mathrm{mm}$ channel; (B) a $3.0-\mathrm{mm}$ ultrathin bronchoscope with a $1.7-\mathrm{mm}$ channel; (C) a 4.0-mm-diameter thin bronchoscope with a 2.0 -mm-diameter channel; (D) a $4.8-\mathrm{mm}$ standard bronchoscope with a $2.0-\mathrm{mm}$-diameter channel; and (E) a $5.9-\mathrm{mm}$ therapeutic bronchoscope with a $3.0-\mathrm{mm}-$ diameter channel.

bronchoscope equipped with a 1.7-mm working channel, which allows the use of radial-probe EBUS (rEBUS), was developed and is now available for use in clinical practice (19-22). We present the following article in accordance with the narrative review checklist (available at http://dx.doi. org/10.21037/jtd-2020-abpd-001).

\section{History}

The idea of using a thinner bronchoscope is not novel. In the early development of flexible bronchoscopes, Shigeto Ikeda, the father of flexible bronchoscopy, manufactured several prototype bronchoscopes of different sizes, including a $3.3-\mathrm{mm}$ thin bronchoscope (23). A few years later, the diameter of the thinnest prototype bronchoscope was reduced to $2.5 \mathrm{~mm}$ (24). In the $1980 \mathrm{~s}$, some thin bronchoscopes equipped with small working channels were developed, mainly for pediatric use. The first publication regarding the usefulness of a thin bronchoscope for PPLs in adult patients was reported by Prakash in 1985 (25). He reported three cases of PPLs in adult patients that could not be observed using a 4.9-mm bronchoscope but were successfully observed using a 3.6- $\mathrm{mm}$ thin bronchoscope. Various types of smallcaliber bronchoscopes have since been developed, and several studies are available on their usefulness in diagnosing PPLs in adult patients $(19,26-30)$. Bronchoscopes with a variety of external diameters and working-channel inner diameters are now available for clinical use (Figure 1).

\section{Techniques}

Although ultrathin bronchoscopes can be advanced close to PPLs, the localization of the target lesion is performed by fluoroscopy and rEBUS and not by direct bronchoscopic vision; thus, these imaging devices are necessary during ultrathin bronchoscopy.

The bronchial route is predicted before procedures by reading a preprocedural high-resolution chest CT scan (31). The anesthetic agents and techniques used are similar to those of standard bronchoscopy. Lidocaine is usually used for topical anesthesia and intravenous midazolam and fentanyl for conscious sedation. Ultrathin bronchoscopy can be performed through either the mouth or the nose. We usually insert a 5.0-mm-inner-diameter tracheal tube transnasally into the trachea. The airway established with the tracheal tube facilitates repeated insertion and removal of the ultrathin bronchoscope, reduces damage from rubbing of the nasal mucosa and vocal cords during bronchoscopy, and reduces deflection of the ultrathin bronchoscope.

After examining the endobronchial region, the ultrathin bronchoscope is advanced into the bronchial route, which is indicated by the navigation device on real-time fluoroscopy. The ultrathin bronchoscope approaches the target lesion and is then localized by rEBUS and fluoroscopy. If the tumor surrounding the EBUS probe is visualized on the EBUS image, the EBUS probe is removed and biopsy forceps are advanced through the same route. We usually perform biopsies under fluoroscopic guidance until 10 visible specimens have been obtained.

\section{Direct observability}

Small-caliber bronchoscopes can be advanced into deeper bronchi than large-caliber bronchoscopes (Figure 2) and, therefore, the possibility of direct observation of a peripheral endobronchial lesion increases with the use of a thin bronchoscope. Rooney et al. reported that 4 of 17 PPLs (24\%) that could not be observed using a $6.3-\mathrm{mm}$ bronchoscope could be observed directly using a $3.3-\mathrm{mm}$ bronchoscope (3). Oki et al. reported that a $3.5-\mathrm{mm}$ bronchoscope could reach two more distal generations of bronchi compared to a 5.9-mm bronchoscope, and 14 of 102 lesions (14\%) were observed only using the $3.5-\mathrm{mm}$ bronchoscope (28). 

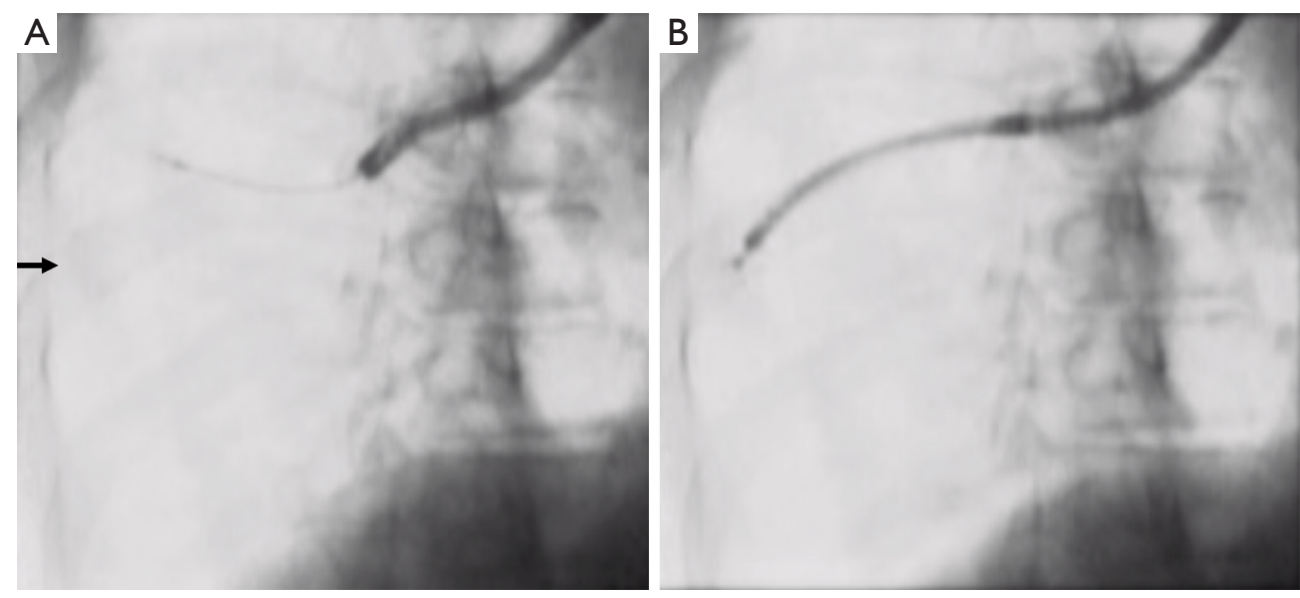

Figure 2 Fluoroscopic images showing the accessibility of a PPL using a 3.0-mm ultrathin bronchoscope and a $4.0-\mathrm{mm}$ bronchoscope. (A) The EBUS probe could not be advanced towards the target lesion (arrow) using a 4.0-mm bronchoscope. (B) The 3.0-mm ultrathin bronchoscope approached the lesion and provided a diagnosis of adenocarcinoma. PPL, peripheral pulmonary lesion; EBUS, endobronchial ultrasound.

\section{Diagnostic yields}

The study results on bronchoscopy using ultrathin bronchoscopes for PPLs are summarized in Table 1. The overall diagnostic yield of ultrathin bronchoscopy is $66 \%$, with a yield of $59 \%$ for lesions $<2 \mathrm{~cm}$. These yields seem comparable to those of other guided bronchoscopy procedures $(5,33)$. As shown in Table 1, ultrathin bronchoscopes have been used with various guiding methods, including rEBUS, navigation devices, fluoroscopy, and CT fluoroscopy. The diagnostic utility of ultrathin bronchoscopes can be enhanced by combining them with other guiding methods.

\section{Randomized trials among bronchoscopes of different sizes}

Several randomized studies comparing diagnostic yields among bronchoscopes of different sizes have been published. Franzen et al. conducted a small pilot study comparing bronchoscopy using a conventional $2.8-\mathrm{mm}$ ultrathin bronchoscope with a $1.2-\mathrm{mm}$ working channel to standardsize bronchoscopes with external diameters of $5.0-6.0 \mathrm{~mm}$ for diagnosing PPLs in a region endemic for tuberculosis (32). Forty patients were enrolled and assigned to either ultrathin or standard-size bronchoscope groups, of whom $28 \%$ were ultimately diagnosed with tuberculosis. The diagnostic yields in the ultrathin bronchoscope group and standardsize bronchoscope group were $55 \%$ and $80 \%(\mathrm{P}=0.95)$, respectively. Adverse events, including extensive coughing, a blocked working channel, and arterial hypertension were more frequent in the ultrathin bronchoscope group. Bronchoscopy times in the ultrathin bronchoscope group and the standard-size bronchoscope group were 31 and $26 \mathrm{~min}$, respectively $(\mathrm{P}=0.15)$. These results fail to show the superiority of fluoroscopy-guided bronchoscopy with a conventional ultrathin bronchoscope over a standardsize bronchoscope. Oki et al. conducted a randomized non-inferiority study of rEBUS-guided bronchoscopy using a 3.4-mm bronchoscope compared to rEBUS with a guide sheath (GS)-guided bronchoscopy using a $4.0-\mathrm{mm}$ bronchoscope (30). In total, 203 patients with PPLs with a median diameter of $26 \mathrm{~mm}$, were analyzed. The diagnostic yields of bronchoscopy using the 3.4-mm and $4.0-\mathrm{mm}$ bronchoscopes were $65 \%$ and $62 \%$, respectively. The difference in diagnostic yield was $3.6 \%$, with a $90 \%$ confidence interval from $-7.5 \%$ to $14.7 \%$. The lower limit of the confidence interval was higher than the predetermined margin of $-10 \%$, thus confirming the non-inferiority of the procedure with the 3.4-mm bronchoscope. Later, Oki et al. conducted a multicenter randomized study comparing rEBUS, fluoroscopy, and virtual bronchoscopic navigation (VBN)-guided bronchoscopy using a 3.0-mm ultrathin bronchoscope to rEBUS-GS, fluoroscopy, and VBNguided bronchoscopy using a 4.0-mm bronchoscope (19). The results in 305 patients with PPLs with a median diameter of $19 \mathrm{~mm}$ were analyzed. The histological 


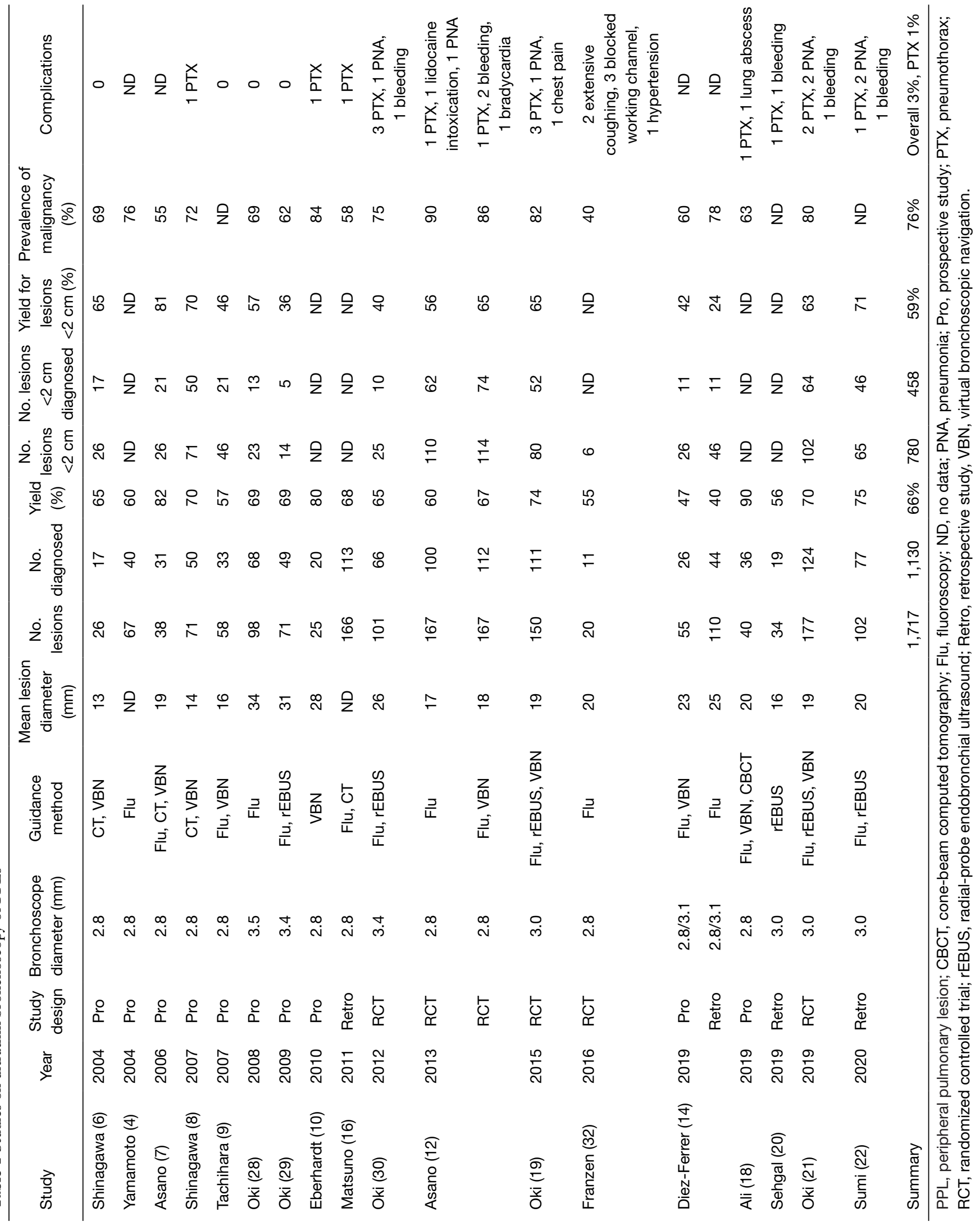


diagnostic yield of multimodality bronchoscopy using the $3.0-\mathrm{mm}$ ultrathin bronchoscope was significantly higher than that with the $4.0-\mathrm{mm}$ bronchoscope ( $74 \%$ vs. $59 \%$, respectively, $\mathrm{P}=0.04)$. The median bronchus level attained using the 3.0-mm-diameter ultrathin bronchoscope was the fifth-generation level, thus more distal than that achieved by the 4.0-mm-diameter bronchoscope (median fourthgeneration) and comparable to that of a conventional $2.8-\mathrm{mm}$ ultrathin bronchoscope [median fifth-generation (12)]. Complications, including pneumothorax, bleeding, chest pain, and pneumonia occurred in $3 \%$ and $5 \%$ of cases in the respective groups $(\mathrm{P}=0.6)$. Oki et al. further performed a randomized study comparing the $3.0-\mathrm{mm}$ ultrathin bronchoscopic method to the $4.0-\mathrm{mm}$ bronchoscopic method, which was modified by adding transbronchial needle aspiration (TBNA) and standard-size biopsy forceps (21). In the 4.0-mm bronchoscope group, TBNA was performed for patients in whom the radial EBUS probe could not be inserted into the target lesion. In addition, the use of 1.5-mm forceps with a GS, standard forceps without a GS, or a combination of the two was permitted in the $4.0-\mathrm{mm}$ bronchoscope group. The results in 356 patients with PPLs with a median diameter of $19 \mathrm{~mm}$ were analyzed. The diagnostic superiority of the $3.0-\mathrm{mm}$ ultrathin bronchoscopic method over the 4.0-mm bronchoscopic method was demonstrated again $(70 \% v s .59 \%$, respectively, $\mathrm{P}=0.03)$. The incidence of complications did not differ between the two groups ( $3 \%$ vs. $5 \%$, respectively, $\mathrm{P}=0.57$ ).

\section{Safety}

As shown in Table 1, the complication rate related to ultrathin bronchoscopy is approximately $3 \%$, and the occurrence of pneumothorax is $1 \%$, which are rates comparable to those of bronchoscopy using larger bronchoscopes (5). Ultrathin bronchoscopes can reach the visceral pleura in certain cases, so they can damage the visceral pleura directly, which causes pneumothorax. Oki et al. reported that pneumothorax occurred in 6 of 410 patients $(1.5 \%)$ who underwent transbronchial forceps biopsy using a $2.8-\mathrm{mm}$ ultrathin bronchoscope under fluoroscopy; four cases were related to the forceps biopsy, and the remaining two were caused by the ultrathin bronchoscope itself (34).

\section{Limitations of ultrathin bronchoscopes}

The obvious disadvantage of a thinner bronchoscope is the limitation of available biopsy instruments. The diagnosis of lung cancer includes genotype as well as subtype classifications, so it is necessary to obtain a sufficient amount of tumor tissue for molecular and morphological analyses. Relatively small $1.5-\mathrm{mm}$ forceps must be used when performing bronchoscopic sampling using an ultrathin bronchoscope with a $1.7-\mathrm{mm}$ working channel. The size of the specimens obtained using $1.5-\mathrm{mm}$ forceps is smaller than those obtained with 1.8 - or 1.9-mm standard forceps. This issue notwithstanding, the $1.5-\mathrm{mm}$ forceps have been widely used not only during ultrathin bronchoscopy but also for bronchoscopy with EBUS-GS, and many investigators have reported a high diagnostic yield of bronchoscopic biopsy using 1.5-mm forceps (19-22,28-30,35-43). Indeed, one study suggested that the size of the biopsy forceps did not affect the diagnostic yield of bronchoscopy (44). In addition, high degrees of concordance of results of genotyping (45), subtyping (46), and programmed deathligand (47) between specimens obtained with $1.5-\mathrm{mm}$ forceps and surgical specimens have been reported.

\section{Future perspectives}

Some promising instruments that can be used during ultrathin bronchoscopy have been developed. Bronchoscopic aspiration needles have recently undergone improvement, and thinner and more flexible needles compared to conventional needles are now available for use in clinical practice (48). A new 21-gauge needle can be used through a 1.7-mm working channel of an ultrathin bronchoscope. Conventional bronchoscopic aspiration needles are stiff, and their steerability and accessibility in the peripheral lung are quite limited (21), while the flexibility of the new needle facilitates TBNA procedures for PPLs (49). The use of TBNA seems to be reasonable in certain cases (e.g., lesions into which rEBUS cannot be inserted), as TBNA can be used to gain access and obtain specimens from peribronchial lesions. The utility of TBNA should be evaluated in terms of efficacy, safety, and cost-effectiveness. Further studies are needed to determine the indications for TBNA.

Another promising instrument is the cryoprobe. Cryobiopsy is an effective diagnostic method for PPLs because it provides larger and better-quality specimens (50). An ultrathin 1.1-mm cryoprobe, which is used through the working channel of an ultrathin bronchoscope, has already been adopted in clinics worldwide. The ultrathin cryoprobe is flexible enough to access PPLs located past the deepcurved bronchus (51). The use of an ultrathin cryoprobe during ultrathin bronchoscopy may overcome the limitation 
of a small sample size.

Bronchoscope manufacturers have continued efforts to develop thinner bronchoscopes with larger working channels and better visibility. In addition, sampling instruments that can be used through the small working channel of an ultrathin bronchoscope have been developed and improved. These efforts will continue in the future and will enhance the diagnostic yield of ultrathin bronchoscopy for PPLs.

\section{Acknowledgments}

Funding: None.

\section{Footnote}

Provenance and Peer Review: This article was commissioned by the Guest Editor (Jiayuan Sun) for the series "Advance in Bronchoscopy for Peripheral Pulmonary Diseases" published in Fournal of Thoracic Disease. The article has undergone external peer review.

Reporting Checklist: The authors have completed the narrative review checklist. Available at http://dx.doi. org/10.21037/jtd-2020-abpd-001

Conflicts of Interest: Both authors have completed the ICMJE uniform disclosure form (available at http://dx.doi. org/10.21037/jtd-2020-abpd-001). The series "Advance in Bronchoscopy for Peripheral Pulmonary Diseases" was commissioned by the editorial office without any funding or sponsorship. The authors have no other conflicts of interest to declare.

Ethical Statement: The authors are accountable for all aspects of the work in ensuring that questions related to the accuracy or integrity of any part of the work are appropriately investigated and resolved.

Open Access Statement: This is an Open Access article distributed in accordance with the Creative Commons Attribution-NonCommercial-NoDerivs 4.0 International License (CC BY-NC-ND 4.0), which permits the noncommercial replication and distribution of the article with the strict proviso that no changes or edits are made and the original work is properly cited (including links to both the formal publication through the relevant DOI and the license). See: https://creativecommons.org/licenses/by-nc-nd/4.0/.

\section{References}

1. Rivera MP, Mehta AC, Wahidi MM. Establishing the diagnosis of lung cancer: Diagnosis and management of lung cancer, 3rd ed: American College of Chest Physicians evidence-based clinical practice guidelines. Chest 2013;143:e142S-65S.

2. Dhillon SS, Harris K. Bronchoscopy for the diagnosis of peripheral lung lesions. J Thorac Dis 2017;9:S1047-58.

3. Rooney CP, Wolf K, McLennan G. Ultrathin bronchoscopy as an adjunct to standard bronchoscopy in the diagnosis of peripheral lung lesions: a preliminary report. Respiration 2002;69:63-8.

4. Yamamoto S, Ueno K, Imamura F, et al. Usefulness of ultrathin bronchoscopy in diagnosis of lung cancer. Lung Cancer 2004;46:43-8.

5. Wang Memoli JS, Nietert PJ, Silvestri GA. Metaanalysis of guided bronchoscopy for the evaluation of the pulmonary nodule. Chest 2012;142:385-93.

6. Shinagawa N, Yamazaki K, Onodera Y, et al. CT-guided transbronchial biopsy using an ultrathin bronchoscope with virtual bronchoscopic navigation. Chest 2004;125:1138-43.

7. Asano F, Matsuno Y, Shinagawa N, et al. A virtual bronchoscopic navigation system for pulmonary peripheral lesions. Chest 2006;130:559-66.

8. Shinagawa N, Yamazaki K, Onodera Y, et al. Virtual bronchoscopic navigation system shortens the examination time--feasibility study of virtual bronchoscopic navigation system. Lung Cancer 2007;56:201-6.

9. Tachihara M, Ishida T, Kanazawa K, et al. A virtual bronchoscopic navigation system under X-ray fluoroscopy for transbronchial diagnosis of small peripheral pulmonary lesions. Lung Cancer 2007;57:322-7.

10. Eberhardt R, Kahn N, Gompelmann D, et al. LungPoint: a new approach to peripheral lesions. J Thorac Oncol 2010;5:1559-63.

11. Iwano S, Imaizumi K, Okada T, et al. Virtual bronchoscopy-guided transbronchial biopsy for aiding the diagnosis of peripheral lung cancer. Eur J Radiol 2011;79:155-9.

12. Asano F, Shinagawa N, Ishida T, et al. Virtual bronchoscopic navigation combined with ultrathin bronchoscopy: a randomized clinical trial. Am J Respir Crit Care Med 2013;188:327-33.

13. Biswas A, Mehta HJ, Sriram PS. Diagnostic yield of the virtual bronchoscopic navigation system guided sampling of peripheral lung lesions using ultrathin bronchoscope and 
protected bronchial brush. Turk Thorac J 2019;20:6-11.

14. Diez-Ferrer M, Morales A, Tebé C, et al. Ultrathin bronchoscopy with and without virtual bronchoscopic navigation: influence of segmentation on diagnostic yield. Respiration 2019;97:252-8.

15. Shinagawa N, Yamazaki K, Onodera Y, et al. Factors related to diagnostic sensitivity using an ultrathin bronchoscope under CT guidance. Chest 2007;131:549-53.

16. Matsuno Y, Asano F, Shindoh J, et al. CT-guided ultrathin bronchoscopy: bioptic approach and factors in predicting diagnosis. Intern Med 2011;50:2143-8.

17. Casal RF, Sarkiss M, Jones AK, et al. Cone beam computed tomography-guided thin/ultrathin bronchoscopy for diagnosis of peripheral lung nodules: a prospective pilot study. J Thorac Dis 2018;10:6950-9.

18. Ali EAA, Takizawa H, Kawakita N, et al. Transbronchial biopsy using an ultrathin bronchoscope guided by conebeam computed tomography and virtual bronchoscopic navigation in the diagnosis of pulmonary nodules. Respiration 2019;98:321-8.

19. Oki M, Saka H, Ando M, et al. Ultrathin bronchoscopy with multimodal devices for peripheral pulmonary lesions: a randomized trial. Am J Respir Crit Care Med 2015;192:468-76.

20. Sehgal IS, Dhooria S, Bal A, et al. A retrospective study comparing the ultrathin versus conventional bronchoscope for performing radial endobronchial ultrasound in the evaluation of peripheral pulmonary lesions. Lung India 2019;36:102-7.

21. Oki M, Saka H, Asano F, et al. Use of an ultrathin vs thin bronchoscope for peripheral pulmonary lesions: a randomized trial. Chest 2019;156:954-64.

22. Sumi T, Ikeda T, Sawai T, et al. Comparison of ultrathin bronchoscopy with conventional bronchoscopy for the diagnosis of peripheral lung lesions without virtual bronchial navigation. Respir Investig 2020;58:376-80.

23. Ikeda S, Yanai N, Ishikawa S. Flexible bronchofiberscope. Keio J Med 1968;17:1-16.

24. Ikeda S, Tsuboi E, Ono R, et al. Flexible bronchofiberscope. Jap J Clin Oncol 1971;1:55-65

25. Prakash UB. The use of the pediatric fiberoptic bronchoscope in adults. Am Rev Respir Dis 1985;132:715-7.

26. Tanaka M, Takizawa H, Satoh M, et al. Assessment of an ultrathin bronchoscope that allows cytodiagnosis of small airways. Chest 1994;106:1443-7.

27. Iyoda A, Suzuki M, Chiyo M, et al. A new thin-type bronchoscope improves diagnostic accuracy of peripheral pulmonary carcinoma. Oncol Rep 2003;10:387-9.

28. Oki M, Saka H, Kitagawa C, et al. Novel thin bronchoscope with a $1.7 \mathrm{~mm}$ working channel for peripheral pulmonary lesions. Eur Respir J 2008;32:465-71.

29. Oki M, Saka H, Kitagawa C, et al. Endobronchial ultrasound-guided transbronchial biopsy using novel thin bronchoscope for diagnosis of peripheral pulmonary lesions. J Thorac Oncol 2009;4:1274-7.

30. Oki M, Saka H, Kitagawa C, et al. Randomized study of endobronchial ultrasound-guided transbronchial biopsy: thin bronchoscopic method versus guide sheath method. J Thorac Oncol 2012;7:535-41.

31. Kurimoto N, Morita K. Bronchial branch tracing. Singapore: Springer, 2020.

32. Franzen D, Diacon AH, Freitag L, et al. Ultrathin bronchoscopy for solitary pulmonary lesions in a region endemic for tuberculosis: a randomised pilot trial. BMC Pulm Med 2016;16:62.

33. Ali MS, Trick W, Mba BI, et al. Radial endobronchial ultrasound for the diagnosis of peripheral pulmonary lesions: a systematic review and meta-analysis. Respirology 2017;22:443-53.

34. Oki M, Saka H, Kitagawa C, et al. Visceral pleural perforation in two cases of ultrathin bronchoscopy. Chest 2005;127:2271-3.

35. Asano F, Matsuno Y, Tsuzuku A, et al. Diagnosis of peripheral pulmonary lesions using a bronchoscope insertion guidance system combined with endobronchial ultrasonography with a guide sheath. Lung Cancer 2008;60:366-73.

36. Ishida T, Asano F, Yamazaki K, et al.; Virtual Navigation in Japan Trial Group. Virtual bronchoscopic navigation combined with endobronchial ultrasound to diagnose small peripheral pulmonary lesions: a randomised trial. Thorax 2011;66:1072-7.

37. Oshige M, Shirakawa T, Nakamura M, et al. Clinical application of virtual bronchoscopic navigation system for peripheral lung lesions. J Bronchology Interv Pulmonol 2011;18:196-202.

38. Tamiya M, Okamoto N, Sasada S, et al. Diagnostic yield of combined bronchoscopy and endobronchial ultrasonography, under LungPoint guidance for small peripheral pulmonary lesions. Respirology 2013;18:834-9.

39. Ikezawa Y, Sukoh N, Shinagawa N, et al. Endobronchial ultrasonography with a guide sheath for pure or mixed ground-glass opacity lesions. Respiration 2014;88:137-43.

40. Steinfort DP, Bonney A, See K, et al. Sequential 
multimodality bronchoscopic investigation of peripheral pulmonary lesions. Eur Respir J 2016;47:607-14.

41. Ikezawa Y, Shinagawa N, Sukoh N, et al. Usefulness of endobronchial ultrasonography with a guide sheath and virtual bronchoscopic navigation for ground-glass opacity lesions. Ann Thorac Surg 2017;103:470-5.

42. Nakai T, Matsumoto Y, Suzuk F, et al. Predictive factors for a successful diagnostic bronchoscopy of ground-glass nodules. Ann Thorac Med 2017;12:171-6.

43. Asano F, Ishida T, Shinagawa N, et al. Virtual bronchoscopic navigation without $\mathrm{X}$-ray fluoroscopy to diagnose peripheral pulmonary lesions: a randomized trial. BMC Pulm Med 2017;17:184.

44. Boonsarngsuk V, Petnak T, So-Ngern A, et al. Comparison of different transbronchial biopsy sampling techniques for the diagnosis of peripheral pulmonary lesions with radial endobronchial ultrasound-guided bronchoscopy: a prospective study. Respir Investig 2020;58:381-6.

45. Oki M, Yatabe Y, Saka H, et al. Feasibility and accuracy of molecular testing in specimens obtained with small biopsy forceps: comparison with the results of surgical specimens. Respiration 2015;89:235-42.

Cite this article as: Oki M, Saka H. Diagnostic value of ultrathin bronchoscopy in peripheral pulmonary lesions: a narrative review. J Thorac Dis 2020;12(12):7675-7682. doi: 10.21037/jtd-2020-abpd-001
46. Takeuchi Y, Shinagawa N, Kikuchi E, et al. The predictive value of endobronchial ultrasonography with a guide sheath in the diagnosis of the histologic subtypes of lung cancer. Respir Investig 2016;54:473-8.

47. Tsunoda A, Morikawa K, Inoue T, et al. A prospective observational study to assess PD-L1 expression in small biopsy samples for non-small-cell lung cancer. BMC Cancer 2019; 19:546.

48. Fielding D, Oki M. Technologies for targeting the peripheral pulmonary nodule including robotics. Respirology 2020;25:914-23.

49. Tremblay A, Myers R, Beaudoin EL, et al. Initial clinical experience with a flexible peripheral 21-G needle device. J Bronchology Interv Pulmonol 2018;25:346-8.

50. Hetzel J, Linzenbold W, Boesmueller H, et al. Evaluation of efficacy of a new cryoprobe for transbronchial cryobiopsy: a randomized, controlled in vivo animal study. Respiration 2020;99:248-56.

51. Kho SS, Chai CS, Nyanti LE, et al. Combination of $1.1 \mathrm{~mm}$ flexible cryoprobe with conventional guide sheath and therapeutic bronchoscope in biopsy of apical upper lobe solitary pulmonary nodule. BMC Pulm Med 2020;20:158. 